\title{
嘧啶核甘-查尔酮杂化体的合成及其抗利什曼原虫活性研究
}

\author{
宰文静 $^{a} \quad$ 蒋盼盼 $^{a} \quad$ 张新迎*,a Philippe M. Loiseau ${ }^{b}$ \\ 郭胜海 ${ }^{a}$ 范学森 ${ }^{*}, a$ \\ $\left({ }^{a}\right.$ 河南师范大学化学化工学院 河南省精细化学品绿色制造协同创新中心 \\ 绿色化学介质与反应省部共建教育部重点实验室 新乡 453007) \\ ( ${ }^{b}$ 法国巴黎第十一大学药学院 沙特奈-马拉布里 F-92290 法国)
}

\begin{abstract}
摘要 以 5-甲酰基嘧啶核苷为原料, 经与(取代)苯乙酮缩合, 得到了一系列含有嘧啶核苷和查尔酮两种优势结构单元 的杂化体. 生物活性研究结果表明, 其中的一些杂化体具有显著的抗利什曼原虫活性, 从而为新药的开发提供了有价 值的先导化合物和构效关系信息.
\end{abstract}

关键词＼cjkstart嘧啶核苷；查尔酮；杂化体；抗利什曼原虫活性

\section{Synthesis and Antileishmanial Activity of Pyrimidine Nucleoside- Chalcone Hybrids}

\author{
Zai, Wenjing $^{a} \quad{\text { Jiang, } \text { Panpan }^{a} \quad \text { Zhang, Xinying }}^{*, a} \quad$ Loiseau, Philippe M. ${ }^{b}$ \\ Guo, Shenghai $^{a} \quad$ Fan, Xuesen ${ }^{*, a}$ \\ $\left({ }^{a}\right.$ Collaborative Innovation Centre of Henan Province for Green Manufacturing of Fine Chemicals, Key Laboratory of Green \\ Chemical Media and Reactions, Ministry of Education, School of Chemistry and Chemical Engineering, \\ Henan Normal University, Xinxiang 453007, China) \\ ( ${ }^{b}$ Chimiothérapie Antiparasitaire, Faculté de Pharmacie, CNRS, UMR 8076 (BioCIS), Université Paris-Sud, 92290 \\ Châtenay-Malabry, France)
}

\begin{abstract}
In this paper, a group of pyrimidine nucleoside-chalcone hybrids were conveniently prepared through condensing 5-formyl pyrimidine nucleosides with (substituted) acetophenones under the promotion of aqueous sodium hydroxide. The hybrid compounds were then evaluated as potential antileishmanial agents and some of them showed promisingly potent activities.

Keywords pyrimidine nucleosides; chalcones; hybrids; antileishmanial activity
\end{abstract}

利什曼病是由利什曼原虫引起的人畜共患寄生虫 病，对人体健康具有严重危害 ${ }^{[1]}$. 该病广泛分布于全世 界的热带和亚热带地区, 患者人数已超过 1 千万, 且近 年来有进一步增加的趋势. 基于这一状况, 世界卫生组 织(WHO) 已将利什曼病列为全球范围的重要健康问 题 ${ }^{[2]}$. 目前, 利什曼病的治疗药物主要有葡萄糖酸锑钠、 葡甲胺锑、两性霉素 $\mathrm{B}$ 、喷他榺、巴龙霉素和米替福新
等 ${ }^{[3]}$. 这些药物尽管大多疗效显著, 但仍存在一定的局 限性，比如具有较大的毒性、价格昂贵、易产生耐药性 等. 因此, 开发新的抗利什曼原虫候选药物对于控制该 病的蔓延具有重要的意义.

研究表明, 对天然核苷的结构改造是发现新先导化 合物的重要途径之一. 在这一领域, 人们相继研究并发 现了多种核苷类抗病毒及抗癌药物 ${ }^{[4,5]}$. 在寻找治疗寄

\footnotetext{
*E-mail: xinyingzhang@htu.cn; xuesen.fan@htu.cn

Received January 14, 2015; revised February 11, 2015; published online February 12, 2015

Project supported by the National Natural Science Foundation of China (Nos. 21172057, 21272058, 21202040), the Program for Science and Technology Innovation Talents in Universities of Henan Province (No. 15HASTIT005), and the Program for Innovative Research Team in Science and Technology in University of Henan Province (No. 15IRTSTHN003).

国家自然科学基金(Nos. 21172057, 21272058, 21202040)、河南省高校科技创新人才支持计划(No. 15HASTIT005)及河南省高校科技创新团队支持计划 (No. 15IRTSTHN003)资助项目.
} 
生虫病药物的过程中, Wataya 和 Loiseau 等 $^{[6]}$ 合成了一 些嘧啶核苷一杂环杂化体类化合物, 并研究了它们的体 外抗利什曼原虫活性, 获得了一些有意义的研究结果; 我们 ${ }^{[7]}$ 在前期的研究工作中, 也合成并发现了一批具有 优异抗利什曼原虫活性的 5-取代嘧啶核苷衍生物. 上述 研究结果表明, 核苷类化合物有可能为利什曼病的治疗 提供具有全新结构特征的候选药物. 另一方面, 许多查 尔酮衍生物均具有显著的抗肿瘤、抗病毒、抗菌、抗溃 疡、抑制和清除氧自由基、抗寄生虫等生物活性. 特别 需要指出的是, Boeck 等 ${ }^{[8]}$ 的研究发现, 查尔酮衍生物不 仅抗寄生虫活性良好, 而且对宿主细胞没有毒性; Liu 等 ${ }^{[9]}$ 发现查尔酮衍生物可以通过抑制在寄生虫呼吸链中 起重要作用的 FRD 酶的活性而显示出抗利什曼原虫无 鞭毛体生长的活性. 鉴于核苷衍生物和查尔酮类化合物 所表现出的良好生物活性, 其中特别是抗利什曼原虫活 性, 我们设计了一种新型的嘧啶核苷-查尔酮杂化体 $(3$, Eq. 1), 期望通过嘧啶核苷与查尔酮这两种药效基团的 结合, 得到活性更好的先导化合物. 所设计的杂化体类 化合物的合成拟以易得的 5-甲酰基嘧啶核苷 1 为原料, 经与(取代)苯乙酮(2)在碱性条件下缩合而得(Eq. 1).

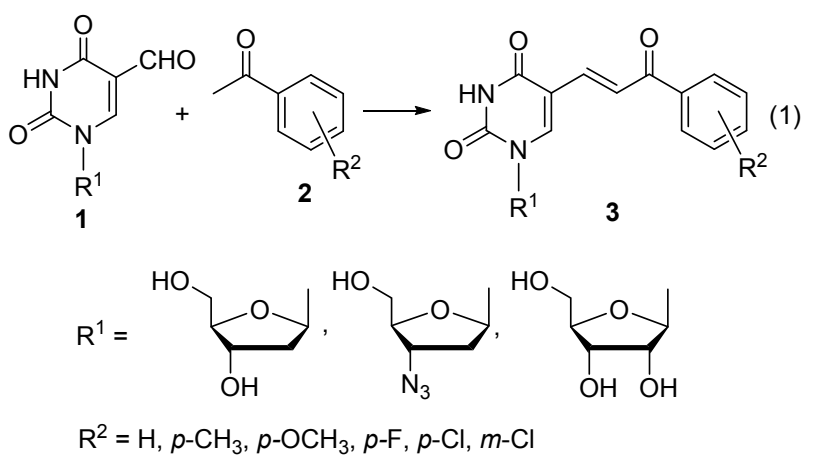

\section{1 结果与讨论}

以 5-甲酰基- $2^{\prime}$-脱氧尿嘧啶核苷(1a)和苯乙酮 (2a) 为 原料, 对所设计的嘧啶核苷一查尔酮杂化体的合成路线 进行了可行性研究(表 1). 将 $\mathbf{1 a}(1 \mathrm{mmol})$ 和 2a (1 mmol) 加入到盛有氢氧化钠 $(1.5 \mathrm{mmol})$ 、水 $(0.5 \mathrm{~mL})$ 和乙醇 $(0.3$ $\mathrm{mL}$ )的反应瓶中, 在 $25{ }^{\circ} \mathrm{C}$ 的温度条件下摚拌反应 $5 \mathrm{~h}$, 然后对反应混合物进行分离纯化. 结果表明, 反应生成 了所期望的 5-(2-苯甲酰基乙烯基)-2'-脱氧尿嘧啶核苷 (3a), 但同时也伴随有副产物 5-(1,3-二苯甲酰基-2-丙 基)- $2^{\prime}$-脱氧尿嘧啶核苷(4a), 两者的收率分别为 $28 \%$ 和 $36 \%$ (表 1, Entry 1). 为提高 3a 的收率, 我们对反应条件 进行了优化. 研究发现, 当反应温度提高时, 有利于 $4 \mathbf{a}$ 的生成(表 1, Entry 2); 而当反应温度降低时, $\mathbf{3 a}$ 的产率 有明显提升. 当反应在 $0{ }^{\circ} \mathrm{C}$ 下进行时, 可以 $68 \%$ 的收率 得到 3a (表 1, Entry 3). 接下来, 又考察了碱的用量对反
应的影响. 结果表明, 碱的用量为 1 equiv.时, 反应速度 较慢(表 1, Entry 4); 随着碱用量的增加, 反应速度加快, 当碱的用量增加到 2 equiv.时, 反应 $2 \mathrm{~h}$ 后产物 $\mathbf{3 a}$ 的收 率可提高至 73\%(表 1, Entry 5); 若继续增加碱的用量, 反应时间虽然可以进一步缩短，但是副产物 $4 \mathrm{a}$ 的比例 会大幅度增加(表 1, Entry 6). 若以氢氧化钾为碱, 则反 应速度较慢, 反应的选择性也较差(表 1, Entry 7)。综合 上述研究结果, 生成 3a 的较佳反应条件是: 氢氧化钠(2 equiv.)为碱、水和乙醇为溶剂、反应温度为 $0{ }^{\circ} \mathrm{C}$ 、反应 时间为 $2 \mathrm{~h}$, 在该条件下, $3 \mathrm{a}$ 的收率为 $73 \%$ (表 1, Entry 5).

表 1 合成 3a 的反应条件优化研究 ${ }^{a}$

Table 1 Optimization study on the preparation of $\mathbf{3 a}$


\begin{tabular}{clcccc}
\hline \multirow{2}{*}{ Entry } & \multirow{2}{*}{ Base (equiv.) } & \multirow{2}{*}{$T /{ }^{\circ} \mathrm{C}$} & \multirow{2}{*}{$t / \mathrm{h}$} & \multicolumn{3}{c}{ Yield $^{b} \%$} \\
\cline { 5 - 6 } & & & & $\mathbf{3 a}$ & $\mathbf{4 a}$ \\
\hline 1 & $\mathrm{NaOH}(1.5)$ & 25 & 5 & 28 & 36 \\
2 & $\mathrm{NaOH}(1.5)$ & 50 & 5 & Trace & 45 \\
3 & $\mathrm{NaOH}(1.5)$ & 0 & 5 & 68 & 10 \\
4 & $\mathrm{NaOH}(1)$ & 0 & 5 & 15 & Trace \\
5 & $\mathrm{NaOH} \mathrm{(2)}$ & 0 & 2 & 73 & 6 \\
6 & $\mathrm{NaOH} \mathrm{(3)}$ & 0 & 1 & 47 & 29 \\
7 & $\mathrm{KOH} \mathrm{(2)}$ & 0 & 5 & 35 & 15 \\
\hline
\end{tabular}

${ }^{a}$ Reaction conditions: $1 \mathrm{mmol}$ of $\mathbf{1 a}, 1 \mathrm{mmol}$ of $\mathbf{2 a}, 0.5 \mathrm{~mL}$ of $\mathrm{H}_{2} \mathrm{O}, 0.3 \mathrm{~mL}$ of ethanol. ${ }^{b}$ Isolated yield.

为考察上述缩合反应的适用范围，同时也为得到更 多的嘧啶核苷一查尔酮杂化体以进行相应的生物活性研 究，我们又尝试了不同种类的 5-甲酰基嘧啶核苷(1)与 苯环上带有不同取代基团的苯乙酮 2 的缩合反应. 表 2 中的数据表明, 5-甲酰基- $2^{\prime}$-脱氧尿嘧啶核苷(1a)、5-甲酰 基-2',3'-双脱氧-3'-叠氮基尿嘧啶核苷(1b)以及 5-甲酰基 尿嘧啶核苷(1c)均是合适的反应底物, 可以与苯环上带 有给电子基团或吸电子基团的苯乙酮(2)发生有效的缩 合反应，生成相应的杂化体 3a $\sim 3 n ，$ 产物的收率良好.

随后，利用溴化噻唑蓝四氮唑(MTT)法对所合成的 嘧啶核苷一查尔酮杂化体抑制杜氏利什曼原虫 (Leishmania donovani)生长活性进行了篎选(表 3). 结果 
表 2 嘧啶核苷一查尔酮杂化体 $\mathbf{3}$ 的制备 ${ }^{a}$

Table 2 Preparation of pyrimidine nucleoside-chalcone hybrids 3<smiles>[R]c1ccc(C(=O)/C=C/c2cn([R1])c(=O)[nH]c2=O)cc1</smiles><smiles>O=C(/C=C/c1cn(C2CC(O)C(CO)O2)c(=O)[nH]c1=O)c1ccccc1</smiles>

3a, $73 \%$<smiles>O=C(/C=C/c1cn(C2CC(O)C(CO)O2)c(=O)[nH]c1=O)c1ccc(F)cc1</smiles>

3d, $50 \%$<smiles>N#CC1CC2OC1CC2n1cc(/C=C/C(=O)c2ccccc2)c(=O)[nH]c1=O</smiles>

$3 g, 65 \%$<smiles></smiles>

3j, 53\%<smiles></smiles><smiles>Cc1ccc(C(=O)/C=C/c2cn(C3CC4OC3COC4CO)c(=O)[nH]c2=O)cc1</smiles>

3b, $75 \%$<smiles>O=C(/C=C/c1cn(C2CC(O)C(CO)O2)c(=O)[nH]c1=O)c1ccc(Cl)cc1</smiles>

3e, $70 \%$<smiles>Cc1ccc(C(=O)/C=C/c2cn(C3CC4CC3OC(CO)C4N)c(=O)[nH]c2=O)cc1</smiles>

3h, $66 \%$<smiles>N#CC1(CO)CC2OC1CC2n1cc(/C=C/C(=O)c2ccc(Cl)cc2)c(=O)[nH]c1=O</smiles>

3k, $64 \%$<smiles>COc1ccc(C(=O)/C=C/c2cn(C3CC4OCC3O4)c(=O)[nH]c2=O)cc1</smiles>

3c, $78 \%$<smiles>O=C(/C=C/c1cn(C2CC(O)C(CO)O2)c(=O)[nH]c1=O)c1cccc(Cl)c1</smiles>

3f, $69 \%$<smiles>COc1ccc(C(=O)/C=C/c2cn(C3CC4(N)CC3OC4CO)c(=O)[nH]c2=O)cc1</smiles>

3i, $68 \%$<smiles>N#CC1CC2OC1COC2n1cc(/C=C/C(=O)c2cccc(Cl)c2)c(=O)[nH]c1=O</smiles>

3I, $70 \%$<smiles>O=C(/C=C/c1cn(C2OC(CO)C(O)C2O)c(=O)[nH]c1=O)c1ccc(Cl)cc1</smiles>

$3 \mathrm{~m}, 63 \%$

3n, $67 \%$

${ }^{a}$ Reaction conditions: $1 \mathrm{mmol}$ of $\mathbf{1}, 1 \mathrm{mmol}$ of $2,2 \mathrm{mmol}$ of $\mathrm{NaOH}, 0.5 \mathrm{~mL}$ of $\mathrm{H}_{2} \mathrm{O}, 0.3 \mathrm{~mL}$ of ethanol, $0{ }^{\circ} \mathrm{C}$; isolated yield.

表明, 在所合成的杂化体化合物中, 3d, 3h, 3j, 3k 和 $3 \mathbf{I}$ 具有显著的抑制杜氏利什曼原虫生长活性，这些结果在 一定程度上支持了通过将嘧啶核苷与查尔酮这两种优 势结构进行杂化可能得到新的抗利什曼原虫先导化合 物的设计思路. 另外, 将所测试化合物的生物活性与其
结构特征进行关联, 初步得到了以下构效关系信息:

杂化体 3h、3k 和 31 均具有显著的抑制杜氏利什曼 原虫生长活性，而杂化体 3b、3e、3f 以及 $3 n$ 均没有活 性，这表明杂化体中嘧啶核苷结构单元中呋喃环的结 构, 特别是 2'-位的脱氧化和 $3^{\prime}$-位取代基的特性可能对 
表 3 嘧啶核苷一查尔酮杂化体 $\mathbf{3}$ 的抗杜氏利什曼原虫活性 $\left[\mathrm{IC}_{50} /\left(\mu \mathrm{mol} \cdot \mathrm{L}^{-1}\right)\right]^{a}$

Table 3 Antileishmanial activities $\left[\mathrm{IC}_{50} /\left(\mu \mathrm{mol} \cdot \mathrm{L}^{-1}\right)\right]$ of nucleoside-chalcone hybrids 3

\begin{tabular}{cc||cc}
\hline Compd. & $\mathrm{IC}_{50} \pm \mathrm{SD}$ & Compd. & $\mathrm{IC}_{50} \pm \mathrm{SD}$ \\
\hline $\mathbf{3 a}$ & $>100$ & $\mathbf{3 i}$ & $>100$ \\
$\mathbf{3 b}$ & $>100$ & $\mathbf{3 j}$ & $16.38 \pm 1.18$ \\
$\mathbf{3 c}$ & $>100$ & $\mathbf{3 k}$ & $6.00 \pm 0.26$ \\
$\mathbf{3 d}$ & $15.02 \pm 1.26$ & $\mathbf{3 l}$ & $7.45 \pm 0.23$ \\
$\mathbf{3 e}$ & $>100$ & $\mathbf{3 m}$ & $>100$ \\
$\mathbf{3 f}$ & $>100$ & $\mathbf{3 n}$ & $>100$ \\
$\mathbf{3 g}$ & $>100$ & $\mathrm{HePC}^{b}$ & $6.72 \pm 0.48$ \\
$\mathbf{3 h}$ & $9.83 \pm 0.81$ & Amphotericin B $^{b}$ & $0.38 \pm 0.08$ \\
\hline
\end{tabular}

${ }^{a} \mathrm{IC}_{50}$ defined as inhibitory concentration $50 \% .{ }^{b}$ Reference compound.

生物活性有着明显的影响.

杂化体 $3 b 、 3 e$ 和 $3 \mathbf{f}$ 没有活性, 而 $3 d$ 具有明显的抑 制活性, 这表明杂化体中查尔酮结构单元的苯环 4-位用 吸电子的氟原子取代可能对于提高生物活性是有利的.

杂化体 $3 \mathbf{h} 、 3 \mathbf{j} 、 3 \mathbf{k}$ 和 31 均具有显著的抑制杜氏利 什曼原虫生长活性, 而杂化体 $\mathbf{3 i}$ 没有活性, 表明杂化体 中查尔酮结构单元的苯环 4-位是强给电子基时可能对 生物活性是不利的.

从上述的构效关系信息可以看出, 杂化体中嘧啶核 苷结构单元中呋喃环上 2'-位的脱氧化、3'-位取代基的特 性以及查尔酮结构单元的芳环电子云密度等因素都对 相应化合物的生物活性有着明显的影响. 在下一阶段的 研究工作中, 我们将在本文研究结果的基础上, 进一步 改造杂化体中核苷结构单元中的呋喃环以及查尔酮结 构单元中的芳环，以期得到活性更高的新先导化合物.

\section{2 结论}

本文以易得的 5-甲酰基嘧啶核苷为原料, 经与(取 代)苯乙酮缩合, 高效合成了一系列嘧啶核苷一查尔酮杂 化体. 通过对该系列化合物的体外抗杜氏利什曼原虫活 性研究, 发现了一些具有显著生物活性的先导化合物; 通过将生物活性与化合物结构特征相关联, 初步得到了 一些有价值的构效关系信息, 为后续的化合物优化及结 构改造打下了基础.

\section{3 实验部分}

\section{1 仪器与试剂}

熔点用 Kofler 显微熔点测定仪测定, 温度计未经校 正; 旋光度用 Chirascan 测定; ${ }^{1} \mathrm{H}$ NMR 及 ${ }^{13} \mathrm{C}$ NMR 使用 Bruker AC 400 核磁共振仪测定, TMS 为内标, DMSO- $d_{6}$ 为溶剂; 红外光谱用 Bruker Vector 22 型红外 分光光度仪测定 $(\mathrm{KBr}$ 压片); 高分辨质谱使用 Bruker microTOF 质谱仪测定.

\section{2 嘧啶核苷一查尔酮杂化体的合成}

向盛有氢氧化钠 $(0.08 \mathrm{~g}, 2 \mathrm{mmol})$ 的反应瓶中加入水 $(0.5 \mathrm{~mL})$ 和乙醇 $(0.3 \mathrm{~mL})$, 然后将该反应瓶置于冰水浴 中冷却, 再加入 5 -甲酰基嘧啶核苷(1, $1 \mathrm{mmol}$ )和(取代) 苯乙酮 $(2,1 \mathrm{mmol})$. 所得混合物在冰水浴中搅拌反应, TLC 跟踪反应. 反应结束后, 旋蒸浓缩, 残留物用二氯 甲烷/甲醇 $(V: V=20: 1)$ 作洗脱剂在硅胶填充柱上进行 柱层析纯化得到嘧啶核苷一查尔酮杂化体 $\mathbf{3}$.

5-(2-苯甲酰基乙烯基)-2'-脱氧尿嘧啶核苷(3a): 浅 黄色固体，m.p. $183 \sim 184{ }^{\circ} \mathrm{C} ;[\alpha]_{\mathrm{D}}^{22}-18.6$ (c 0.10 , EtOH); ${ }^{1} \mathrm{H}$ NMR (DMSO- $\left.d_{6}, 400 \mathrm{MHz}\right) \delta: 2.18 \sim 2.24(\mathrm{~m}$, $\left.2 \mathrm{H}, \mathrm{CH}_{2}\right), 3.59 \sim 3.71\left(\mathrm{~m}, 2 \mathrm{H}, \mathrm{CH}_{2}\right), 3.80 \sim 3.83(\mathrm{~m}, 1 \mathrm{H}$, $\mathrm{CH}), 4.27 \sim 4.29(\mathrm{~m}, 1 \mathrm{H}, \mathrm{CH}), 5.27 \sim 5.31(\mathrm{~m}, 2 \mathrm{H}$, $\mathrm{OH} \times 2), 6.15(\mathrm{t}, J=6.4 \mathrm{~Hz}, 1 \mathrm{H}, \mathrm{CH}), 7.49 \sim 7.67(\mathrm{~m}, 4 \mathrm{H}$, $3 \mathrm{ArH}, \mathrm{CH}), 7.95$ (d, $J=7.2 \mathrm{~Hz}, 2 \mathrm{H}, 2 \mathrm{ArH}), 8.08$ (d, $J=$ $15.6 \mathrm{~Hz}, 1 \mathrm{H}, \mathrm{CH}), 8.59$ (s, 1H, CH), 11.74 (s, 1H, NH); ${ }^{13} \mathrm{C}$ NMR (DMSO- $\left.d_{6}, 100 \mathrm{MHz}\right) \delta: 61.2,70.1,85.5,88.2$, $109.2,120.6,128.5,129.4,133.4,138.3,145.1,149.7$, 162.3, 189.8; IR (KBr) v: 3413, 1689, 1599, 1587, 1466, 1293, 1091, $697 \mathrm{~cm}^{-1}$. HRMS calcd for $\mathrm{C}_{18} \mathrm{H}_{19} \mathrm{~N}_{2} \mathrm{O}_{6}[\mathrm{M}+$ $\mathrm{H}]^{+}:$359.1243, found 359.1254 .

5-[2-(4-甲基苯甲酰基)乙烯基]-2'-脱氧尿嘧啶核苷 (3b): 无色固体, m.p. $189 \sim 191{ }^{\circ} \mathrm{C} ;[\alpha]_{\mathrm{D}}^{22}-22.9$ (c $0.10, \mathrm{EtOH}) ;{ }^{1} \mathrm{H}$ NMR (DMSO- $\left.d_{6}, 400 \mathrm{MHz}\right) \delta: 2.20 \sim$ $2.24\left(\mathrm{~m}, 2 \mathrm{H}, \mathrm{CH}_{2}\right), 2.39\left(\mathrm{~s}, 3 \mathrm{H}, \mathrm{CH}_{3}\right), 3.58 \sim 3.71(\mathrm{~m}, 2 \mathrm{H}$, $\left.\mathrm{CH}_{2}\right), 3.80 \sim 3.82(\mathrm{~m}, 1 \mathrm{H}, \mathrm{CH}), 4.27 \sim 4.29(\mathrm{~m}, 1 \mathrm{H}, \mathrm{CH})$, $5.27 \sim 5.30(\mathrm{~m}, 2 \mathrm{H}, \mathrm{OH} \times 2), 6.15(\mathrm{t}, J=6.4 \mathrm{~Hz}, 1 \mathrm{H}, \mathrm{CH})$, 7.37 (d, $J=7.6 \mathrm{~Hz}, 2 \mathrm{H}, 2 \mathrm{ArH}), 7.49$ (d, $J=15.6 \mathrm{~Hz}, 1 \mathrm{H}$, $\mathrm{CH}), 7.87$ (d, $J=8.0 \mathrm{~Hz}, 2 \mathrm{H}, 2 \mathrm{ArH}), 8.07$ (d, $J=15.6 \mathrm{~Hz}$, $1 \mathrm{H}, \mathrm{CH}), 8.57$ (s, 1H, CH), 11.73 (s, 1H, NH); ${ }^{13} \mathrm{C} \mathrm{NMR}$ (DMSO- $\left.d_{6}, 100 \mathrm{MHz}\right) \delta: 21.6,61.2,70.1,85.4,88.2$, $109.3,120.6,128.7,129.9,135.8,137.9,143.8,144.9$, 149.7, 162.3, 189.2; IR (KBr) v: 3412, 1703, 1609, 1584, 1467, 1298, 1091, $823 \mathrm{~cm}^{-1}$. HRMS calcd for $\mathrm{C}_{19} \mathrm{H}_{21} \mathrm{~N}_{2} \mathrm{O}_{6}$ $[\mathrm{M}+\mathrm{H}]^{+}: 373.1400$, found 373.1412 .

5-[2-(4-甲氧基苯甲酰基)乙烯基]-2'-脱氧尿嘧啶核 苷(3c): 黄色固体, m.p. $195 \sim 197{ }^{\circ} \mathrm{C} ;[\alpha]_{\mathrm{D}}^{22}-19.1$ (c $0.10, \mathrm{EtOH}) ;{ }^{1} \mathrm{H}$ NMR (DMSO- $\left.d_{6}, 400 \mathrm{MHz}\right) \delta: 2.20 \sim$ $2.24\left(\mathrm{~m}, 2 \mathrm{H}, \mathrm{CH}_{2}\right), 3.62 \sim 3.69\left(\mathrm{~m}, 2 \mathrm{H}, \mathrm{CH}_{2}\right), 3.83 \sim 3.86$ $\left(\mathrm{m}, 4 \mathrm{H}, \mathrm{CH}, \mathrm{CH}_{3}\right), 4.28 \sim 4.29(\mathrm{~m}, 1 \mathrm{H}, \mathrm{CH}), 5.23 \sim 5.26$ $(\mathrm{m}, 2 \mathrm{H}, \mathrm{OH} \times 2), 6.16(\mathrm{t}, J=5.6 \mathrm{~Hz}, 1 \mathrm{H}, \mathrm{CH}), 7.09(\mathrm{~d}, J=$ $8.4 \mathrm{~Hz}, 2 \mathrm{H}, 2 \mathrm{ArH}), 7.47$ (d, $J=15.2 \mathrm{~Hz}, 1 \mathrm{H}, \mathrm{CH}), 7.96$ (d, $J=8.4 \mathrm{~Hz}, 2 \mathrm{H}, 2 \mathrm{ArH}), 8.06(\mathrm{~d}, J=15.6 \mathrm{~Hz}, 1 \mathrm{H}, \mathrm{ArH})$, $8.56(\mathrm{~s}, 1 \mathrm{H}, \mathrm{CH}), 11.66$ (br s, $1 \mathrm{H}, \mathrm{NH}) ;{ }^{13} \mathrm{C} \mathrm{NMR}$ $\left(\mathrm{DMSO}-d_{6}, 100 \mathrm{MHz}\right) \delta$ : 56.0, 61.2, 70.1, 85.4, 88.2, 
$109.4,114.6,120.5,130.9,131.1,137.4,144.6,149.8$, 162.4, 163.5, 188.0; IR (KBr) v: 3409, 1702, 1603, 1583, 1466, 1298, 1090, $839 \mathrm{~cm}^{-1}$. HRMS calcd for $\mathrm{C}_{19} \mathrm{H}_{21} \mathrm{~N}_{2} \mathrm{O}_{7}$ $[\mathrm{M}+\mathrm{H}]^{+}: 389.1349$, found 389.1361 .

5-[2-(4-氟苯甲酰基) 乙烯基]-2'-脱氧尿嘧啶核苷 (3d): 黄色固体, m.p. $169 \sim 171{ }^{\circ} \mathrm{C} ;[\alpha]_{\mathrm{D}}^{22}-31.2$ (c $0.10, \mathrm{EtOH}) ;{ }^{1} \mathrm{H}$ NMR (DMSO- $\left.d_{6}, 400 \mathrm{MHz}\right) \delta: 2.19 \sim$ $2.24\left(\mathrm{~m}, 2 \mathrm{H}, \mathrm{CH}_{2}\right), 3.59 \sim 3.71\left(\mathrm{~m}, 2 \mathrm{H}, \mathrm{CH}_{2}\right), 3.82 \sim 3.83$ $(\mathrm{m}, 1 \mathrm{H}, \mathrm{CH}), 4.28 \sim 4.29(\mathrm{~m}, 1 \mathrm{H}, \mathrm{CH}), 5.23 \sim 5.27(\mathrm{~m}, 2 \mathrm{H}$, $\mathrm{OH} \times 2), 6.15(\mathrm{t}, J=6.0 \mathrm{~Hz}, 1 \mathrm{H}, \mathrm{CH}), 7.39$ (t, $J=8.4 \mathrm{~Hz}$, $2 \mathrm{H}, 2 \mathrm{ArH}), 7.51(\mathrm{~d}, J=15.2 \mathrm{~Hz}, 1 \mathrm{H}, \mathrm{CH}), 8.03 \sim 8.06(\mathrm{~m}$, $3 \mathrm{H}, 2 \mathrm{ArH}, \mathrm{CH}), 8.58(\mathrm{~s}, 1 \mathrm{H}, \mathrm{CH}), 11.71(\mathrm{~s}, 1 \mathrm{H}, \mathrm{NH}) ;{ }^{13} \mathrm{C}$ NMR (DMSO- $\left.d_{6}, 100 \mathrm{MHz}\right) \delta: 61.2,70.1,85.5,88.2$, $109.2,116.3,116.5,120.3,131.4,131.5,135.0,138.6$, 145.2, 149.7, 162.3, 164.1, 188.3; IR (KBr) v: 3398, 1694, 1598, 1574, 1466, 1295, 1094, $839 \mathrm{~cm}^{-1}$. HRMS calcd for $\mathrm{C}_{18} \mathrm{H}_{18} \mathrm{FN}_{2} \mathrm{O}_{6}[\mathrm{M}+\mathrm{H}]^{+}: 377.1149$, found 377.1141 .

5-[2-(4-氯苯甲酰基) 乙烯基]-2'-脱氧尿嘧啶核苷 (3e): 黄色固体, m.p. $187 \sim 189{ }^{\circ} \mathrm{C} ;[\alpha]_{\mathrm{D}}^{22}-26.6$ (c $0.10, \mathrm{EtOH}) ;{ }^{1} \mathrm{H}$ NMR (DMSO- $\left.d_{6}, 400 \mathrm{MHz}\right) \delta: 2.16 \sim$ $2.26\left(\mathrm{~m}, 2 \mathrm{H}, \mathrm{CH}_{2}\right), 3.58 \sim 3.69\left(\mathrm{~m}, 2 \mathrm{H}, \mathrm{CH}_{2}\right), 3.80 \sim 3.82$ $(\mathrm{m}, 1 \mathrm{H}, \mathrm{CH}), 4.26 \sim 4.28(\mathrm{~m}, 1 \mathrm{H}, \mathrm{CH}), 5.19(\mathrm{t}, J=5.2 \mathrm{~Hz}$, $1 \mathrm{H}, \mathrm{OH}), 5.23(\mathrm{~d}, J=4.0 \mathrm{~Hz}, 1 \mathrm{H}, \mathrm{OH}), 6.13(\mathrm{t}, J=6.4 \mathrm{~Hz}$, $1 \mathrm{H}, \mathrm{CH}), 7.50$ (d, $J=15.2 \mathrm{~Hz}, 1 \mathrm{H}, \mathrm{CH}), 7.61$ (d, $J=8.4$ $\mathrm{Hz}, 2 \mathrm{H}, 2 \mathrm{ArH}), 7.94$ (d, J=8.4 Hz, 2H, 2ArH), 8.01 (d, $J=15.6 \mathrm{~Hz}, 1 \mathrm{H}, \mathrm{CH}), 8.56$ (s, 1H, CH), 11.67 (s, 1H, NH); ${ }^{13} \mathrm{C}$ NMR (DMSO- $\left.d_{6}, 100 \mathrm{MHz}\right) \delta: 61.2,70.1,85.5,88.2$, $109.1,120.2,129.4,130.3,137.0,138.2,138.8,145.3$, 149.7, 162.2, 188.7; IR (KBr) v: 3349, 1696, 1592, 1468, 1297, 1093, $834 \mathrm{~cm}^{-1}$. HRMS calcd for $\mathrm{C}_{18} \mathrm{H}_{18} \mathrm{ClN}_{2} \mathrm{O}_{6}$ $[\mathrm{M}+\mathrm{H}]^{+}:$393.0853, found 393.0859.

5-[2-(3-氯苯甲酰基)乙烯基]-2'-脱氧尿嘧啶核苷 (3f): 无色固体, m.p. $221 \sim 222{ }^{\circ} \mathrm{C}$; $[\alpha]_{\mathrm{D}}^{22}-37.3$ (c $0.10, \mathrm{EtOH}) ;{ }^{1} \mathrm{H}$ NMR (DMSO- $\left.d_{6}, 400 \mathrm{MHz}\right) \delta: 2.16 \sim$ $2.26\left(\mathrm{~m}, 2 \mathrm{H}, \mathrm{CH}_{2}\right), 3.57 \sim 3.70\left(\mathrm{~m}, 2 \mathrm{H}, \mathrm{CH}_{2}\right), 3.80 \sim 3.81$ $(\mathrm{m}, 1 \mathrm{H}, \mathrm{CH}), 4.25 \sim 4.28(\mathrm{~m}, 1 \mathrm{H}, \mathrm{CH}), 5.19(\mathrm{t}, J=5.2 \mathrm{~Hz}$, $1 \mathrm{H}, \mathrm{OH}), 5.23(\mathrm{~d}, J=4.4 \mathrm{~Hz}, 1 \mathrm{H}, \mathrm{OH}), 6.13(\mathrm{t}, J=6.4 \mathrm{~Hz}$, $1 \mathrm{H}, \mathrm{CH}), 7.51(\mathrm{~d}, J=15.2 \mathrm{~Hz}, 1 \mathrm{H}, \mathrm{CH}), 7.59(\mathrm{t}, J=8.0 \mathrm{~Hz}$, $1 \mathrm{H}, \operatorname{ArH}), 7.71(\mathrm{~d}, J=8.4 \mathrm{~Hz}, 1 \mathrm{H}, \mathrm{ArH}), 7.87 \sim 7.89(\mathrm{~m}$, 2H, 2ArH), 7.99 (d, J=15.6 Hz, 1H, CH), 8.58 (s, 1H, $\mathrm{CH}), 11.69(\mathrm{~s}, 1 \mathrm{H}, \mathrm{NH}) ;{ }^{13} \mathrm{C}$ NMR (DMSO- $\left.d_{6}, 100 \mathrm{MHz}\right)$ $\delta: 61.2,70.1,85.6,88.2,109.1,120.1,127.1,128.0,131.3$, $133.0,134.2,139.2,140.2,145.4,149.7,162.2,188.5$; IR (KBr) v: 3392, 1716, 1686, 1595, 1580, 1467, 1293, 1101, $716 \mathrm{~cm}^{-1}$. HRMS calcd for $\mathrm{C}_{18} \mathrm{H}_{18} \mathrm{ClN}_{2} \mathrm{O}_{6}[\mathrm{M}+\mathrm{H}]^{+}$:
393.0853, found 393.0862.

5-(2-苯甲酰基乙烯基)-2', 3'-双脱氧-3'-叠氮基尿嘧 啶核苷 $(3 g)$ : 无色固体, m.p. $182 \sim 183{ }^{\circ} \mathrm{C} ;[\alpha]_{\mathrm{D}}^{22}-$ 147.0 (c 0.10, EtOH); ${ }^{1} \mathrm{H}$ NMR (DMSO- $\left.d_{6}, 400 \mathrm{MHz}\right) \delta$ : $2.34 \sim 2.41\left(\mathrm{~m}, 1 \mathrm{H}, \mathrm{CH}_{2}-1\right), 2.52 \sim 2.56\left(\mathrm{~m}, 1 \mathrm{H}, \mathrm{CH}_{2}-2\right)$, $3.61 \sim 3.65\left(\mathrm{~m}, 1 \mathrm{H}, \mathrm{CH}_{2}-1\right), 3.73 \sim 3.76\left(\mathrm{~m}, 1 \mathrm{H}, \mathrm{CH}_{2}-2\right)$, $3.84 \sim 3.86(\mathrm{~m}, 1 \mathrm{H}, \mathrm{CH}), 4.41 \sim 4.46(\mathrm{~m}, 1 \mathrm{H}, \mathrm{CH}), 5.45(\mathrm{t}$, $J=4.8 \mathrm{~Hz}, 1 \mathrm{H}, \mathrm{OH}), 6.08(\mathrm{t}, J=5.2 \mathrm{~Hz}, 1 \mathrm{H}, \mathrm{CH}), 7.49 \sim$ 7.57 (m, 3H, 2ArH, CH), $7.64(\mathrm{t}, J=7.2 \mathrm{~Hz}, 1 \mathrm{H}, \mathrm{ArH})$, 7.94 (d, $J=7.6 \mathrm{~Hz}, 2 \mathrm{H}, 2 \mathrm{ArH}), 8.06$ (d, $J=15.2 \mathrm{~Hz}, 1 \mathrm{H}$, $\mathrm{CH}), 8.56(\mathrm{~s}, 1 \mathrm{H}, \mathrm{CH}), 11.74(\mathrm{~s}, 1 \mathrm{H}, \mathrm{NH}) ;{ }^{13} \mathrm{C} \mathrm{NMR}$ $\left(\mathrm{DMSO}-d_{6}, 100 \mathrm{MHz}\right) \delta: 37.4,59.3,60.5,85.0,109.2$, $120.6,128.5,129.3,133.3,138.3,145.0,149.6,162.3$, 189.7; IR (KBr) v: 3375, 2088, 1722, 1686, 1585, 1567, 1460, 1290, 1092, $687 \mathrm{~cm}^{-1}$. HRMS calcd for $\mathrm{C}_{18} \mathrm{H}_{18} \mathrm{~N}_{5} \mathrm{O}_{5}$ $[\mathrm{M}+\mathrm{H}]^{+}:$384.1308, found 384.1319.

5-[2-(4-甲基苯甲酰基)乙烯基]-2',3'-双脱氧-3'-叠氮 基尿嘧啶核苷(3h): 无色固体, m.p. 199 201 ${ }^{\circ} \mathrm{C}$; $[\alpha]_{\mathrm{D}}^{22}$ -138.0 (c 0.10, EtOH); ${ }^{1} \mathrm{H}$ NMR (DMSO- $\left.d_{6}, 400 \mathrm{MHz}\right) \delta$ : $2.34 \sim 2.37\left(\mathrm{~m}, 4 \mathrm{H}, \mathrm{CH}_{3}, \mathrm{CH}_{2}-1\right), 2.48 \sim 2.53(\mathrm{~m}, 1 \mathrm{H}$, $\left.\mathrm{CH}_{2}-2\right), 3.60 \sim 3.64\left(\mathrm{~m}, 1 \mathrm{H}, \mathrm{CH}_{2}-1\right), 3.72 \sim 3.75(\mathrm{~m}, 1 \mathrm{H}$, $\left.\mathrm{CH}_{2}-2\right), 3.84 \sim 3.86(\mathrm{~m}, 1 \mathrm{H}, \mathrm{CH}), 4.43(\mathrm{q}, J=6.4 \mathrm{~Hz}, 1 \mathrm{H}$, $\mathrm{CH}), 5.42(\mathrm{t}, J=4.8 \mathrm{~Hz}, 1 \mathrm{H}, \mathrm{OH}), 6.08(\mathrm{t}, J=5.2 \mathrm{~Hz}, 1 \mathrm{H}$, $\mathrm{CH}), 7.35$ (d, $J=7.6 \mathrm{~Hz}, 2 \mathrm{H}, 2 \mathrm{ArH}), 7.48$ (d, $J=15.6 \mathrm{~Hz}$, $1 \mathrm{H}, \mathrm{CH}), 7.85$ (d, $J=8.0 \mathrm{~Hz}, 2 \mathrm{H}, 2 \mathrm{ArH}), 8.05$ (d, $J=15.2$ $\mathrm{Hz}, 1 \mathrm{H}, \mathrm{CH}), 8.53(\mathrm{~s}, 1 \mathrm{H}, \mathrm{CH}), 11.72(\mathrm{~s}, 1 \mathrm{H}, \mathrm{NH}) ;{ }^{13} \mathrm{C}$ NMR (DMSO- $\left.d_{6}, 100 \mathrm{MHz}\right) \delta: 21.6,37.4,59.3,60.5,85.0$, $109.3,120.7,128.6,129.8,135.7,137.8,143.7,144.8$, 149.6, 162.3, 189.2; IR (KBr) v: 3430, 2100, 1715, 1687, 1587, 1464, 1292, 1091, $826 \mathrm{~cm}^{-1}$. HRMS calcd for $\mathrm{C}_{19} \mathrm{H}_{20} \mathrm{~N}_{5} \mathrm{O}_{5}[\mathrm{M}+\mathrm{H}]^{+}: 398.1464$, found 398.1458.

5-[2-(4-甲氧基苯甲酰基)乙烯基]-2',3'-双脱氧-3'-叠 氮基尿嘧啶核苷(3i): 无色固体, m.p. 200 202 ${ }^{\circ} \mathrm{C}$; $[\alpha]_{\mathrm{D}}^{22}-91.9$ (c 0.10, EtOH); ${ }^{1} \mathrm{H}$ NMR (DMSO- $d_{6}, 400$ $\mathrm{MHz}) \delta: 2.18 \sim 2.24\left(\mathrm{~m}, 1 \mathrm{H}, \mathrm{CH}_{2}-1\right), 2.69 \sim 2.76(\mathrm{~m}, 1 \mathrm{H}$, $\left.\mathrm{CH}_{2}-2\right), 3.50 \sim 3.52\left(\mathrm{~m}, 2 \mathrm{H}, \mathrm{CH}_{2}\right), 3.84\left(\mathrm{~s}, 3 \mathrm{H}, \mathrm{CH}_{3}\right)$, $4.35 \sim 4.37(\mathrm{~m}, 1 \mathrm{H}, \mathrm{CH}), 4.47 \sim 4.49(\mathrm{~m}, 1 \mathrm{H}, \mathrm{CH}), 5.02(\mathrm{t}$, $J=5.6 \mathrm{~Hz}, 1 \mathrm{H}, \mathrm{OH}), 6.02 \sim 6.04(\mathrm{~m}, 1 \mathrm{H}, \mathrm{CH}), 7.07(\mathrm{~d}, J=$ $8.8 \mathrm{~Hz}, 2 \mathrm{H}, 2 \mathrm{ArH}), 7.56$ (d, $J=15.2 \mathrm{~Hz}, 1 \mathrm{H}, \mathrm{CH}), 7.95$ (d, $J=8.4 \mathrm{~Hz}, 2 \mathrm{H}, 2 \mathrm{ArH}), 8.12$ (d, $J=15.6 \mathrm{~Hz}, 1 \mathrm{H}, \mathrm{CH}), 8.34$ $(\mathrm{s}, 1 \mathrm{H}, \mathrm{CH}), 11.67$ (br s, $1 \mathrm{H}, \mathrm{NH}) ;{ }^{13} \mathrm{C} \mathrm{NMR}$ (DMSO- $d_{6}$, $100 \mathrm{MHz}) \delta$ : 37.6, 55.9, 61.0, 61.7, 86.4, 87.2, 109.0, $114.5,120.7,130.8,131.1,137.8,145.0,149.6,162.5$, 163.4, 188.2; IR (KBr) v: 3401, 2098, 1713, 1686, 1604, 1586, 1464, 1293, 1090, $838 \mathrm{~cm}^{-1}$. HRMS calcd for 
$\mathrm{C}_{19} \mathrm{H}_{20} \mathrm{~N}_{5} \mathrm{O}_{6}[\mathrm{M}+\mathrm{H}]^{+}:$414.1414, found 414.1407.

5-[2-(4-氟苯甲酰基)乙烯基]-2',3'-双脱氧-3'-叠氮基 尿嘧啶核苷 $(3 \mathbf{j})$ : 无色固体, m.p. $185 \sim 186{ }^{\circ} \mathrm{C} ;[\alpha]_{\mathrm{D}}^{22}-$ 122.8 (c 0.10, EtOH); ${ }^{1} \mathrm{H}$ NMR (DMSO- $\left.d_{6}, 400 \mathrm{MHz}\right) \delta$ : $2.34 \sim 2.41\left(\mathrm{~m}, 1 \mathrm{H}, \mathrm{CH}_{2}-1\right), 2.48 \sim 2.56\left(\mathrm{~m}, 1 \mathrm{H}, \mathrm{CH}_{2}-2\right)$, $3.61 \sim 3.66\left(\mathrm{~m}, 1 \mathrm{H}, \mathrm{CH}_{2}-1\right), 3.72 \sim 3.77\left(\mathrm{~m}, 1 \mathrm{H}, \mathrm{CH}_{2}-2\right)$, $3.85 \sim 3.88(\mathrm{~m}, 1 \mathrm{H}, \mathrm{CH}), 4.43(\mathrm{q}, J=6.8 \mathrm{~Hz}, 1 \mathrm{H}, \mathrm{CH})$, $5.39(\mathrm{t}, J=5.2 \mathrm{~Hz}, 1 \mathrm{H}, \mathrm{OH}), 6.07 \sim 6.10(\mathrm{~m}, 1 \mathrm{H}, \mathrm{CH}), 7.37$ (t, $J=8.8 \mathrm{~Hz}, 2 \mathrm{H}, 2 \mathrm{ArH}), 7.50$ (d, $J=15.6 \mathrm{~Hz}, 1 \mathrm{H}, \mathrm{CH})$, $8.00 \sim 8.06(\mathrm{~m}, 3 \mathrm{H}, 2 \mathrm{ArH}, \mathrm{CH}), 8.54(\mathrm{~s}, 1 \mathrm{H}, \mathrm{CH}), 11.70(\mathrm{~s}$, $1 \mathrm{H}, \mathrm{NH}) ;{ }^{13} \mathrm{C}$ NMR (DMSO-d, $\left.100 \mathrm{MHz}\right) \delta: 37.4,59.4$, 60.6, 85.1, 109.2, 116.2, 116.4, 120.4, 131.3, 131.4, 135.0, $138.4,145.0,149.6,162.2,164.0,166.5,188.3$; IR (KBr) v: 3430, 2109, 1717, 1687, 1600, 1575, 1466, 1294, 1096, $842 \mathrm{~cm}^{-1}$. HRMS calcd for $\mathrm{C}_{18} \mathrm{H}_{17} \mathrm{FN}_{5} \mathrm{O}_{5}[\mathrm{M}+\mathrm{H}]^{+}$: 402.1214, found 402.1226 .

5-[2-(4-氯苯甲酰基)乙烯基]-2',3'-双脱氧-3'-叠氮基 尿嘧啶核苷(3k): 无色固体，m.p. $198 \sim 199{ }^{\circ} \mathrm{C} ;[\alpha]_{\mathrm{D}}^{22}$ -130.8 (c 0.10, EtOH); ${ }^{1} \mathrm{H}$ NMR (DMSO- $\left.d_{6}, 400 \mathrm{MHz}\right) \delta$ : $2.35 \sim 2.41\left(\mathrm{~m}, 1 \mathrm{H}, \mathrm{CH}_{2}-1\right), 2.48 \sim 2.53\left(\mathrm{~m}, 1 \mathrm{H}, \mathrm{CH}_{2}-2\right)$, $3.60 \sim 3.65\left(\mathrm{~m}, 1 \mathrm{H}, \mathrm{CH}_{2}-1\right), 3.72 \sim 3.76\left(\mathrm{~m}, 1 \mathrm{H}, \mathrm{CH}_{2}-2\right)$, $3.85 \sim 3.87(\mathrm{~m}, 1 \mathrm{H}, \mathrm{CH}), 4.43$ (q, $J=6.4 \mathrm{~Hz}, 1 \mathrm{H}, \mathrm{CH})$, 5.39 (t, $J=5.2 \mathrm{~Hz}, 1 \mathrm{H}, \mathrm{OH}), 6.07$ (t, $J=5.2 \mathrm{~Hz}, 1 \mathrm{H}, \mathrm{CH})$, $7.51(\mathrm{~d}, J=15.2 \mathrm{~Hz}, 1 \mathrm{H}, \mathrm{CH}), 7.62$ (d, $J=8.4 \mathrm{~Hz}, 2 \mathrm{H}$, 2ArH), 7.94 (d, $J=8.4 \mathrm{~Hz}, 2 \mathrm{H}, 2 \mathrm{ArH}), 8.02$ (d, $J=15.6$ $\mathrm{Hz}, 1 \mathrm{H}, \mathrm{CH}), 8.54$ (s, 1H, CH), $11.71(\mathrm{~s}, 1 \mathrm{H}, \mathrm{NH}) ;{ }^{13} \mathrm{C}$ NMR (DMSO- $\left.d_{6}, 100 \mathrm{MHz}\right) \delta:$ 37.4, 59.4, 60.6, 85.1, $109.1,120.3,129.4,130.3,136.9,138.2,138.8,145.2$, 149.6, 162.2, 188.7; IR (KBr) v: 3452, 2107, 1720, 1688, 1593, 1467, 1293, 1093, $834 \mathrm{~cm}^{-1}$. HRMS calcd for $\mathrm{C}_{18} \mathrm{H}_{17} \mathrm{ClN}_{5} \mathrm{O}_{5}[\mathrm{M}+\mathrm{H}]^{+}:$418.0918, found 418.0907.

5-[2-(3-氯苯甲酰基)乙烯基]-2',3'-双脱氧-3'-叠氮基 尿嘧啶核苷(3l): 无色固体, m.p. $150 \sim 152{ }^{\circ} \mathrm{C} ;[\alpha]_{\mathrm{D}}^{22}-$ 118.1 (c 0.10, EtOH); ${ }^{1} \mathrm{H}$ NMR (DMSO- $\left.d_{6}, 400 \mathrm{MHz}\right) \delta$ : $2.36 \sim 2.43\left(\mathrm{~m}, 1 \mathrm{H}, \mathrm{CH}_{2}-1\right), 2.50 \sim 2.58\left(\mathrm{~m}, 1 \mathrm{H}, \mathrm{CH}_{2}-2\right)$, $3.62 \sim 3.67\left(\mathrm{~m}, 1 \mathrm{H}, \mathrm{CH}_{2}-1\right), 3.73 \sim 3.78\left(\mathrm{~m}, 1 \mathrm{H}, \mathrm{CH}_{2}-2\right)$, $3.85 \sim 3.88(\mathrm{~m}, 1 \mathrm{H}, \mathrm{CH}), 4.45(\mathrm{q}, J=6.4 \mathrm{~Hz}, 1 \mathrm{H}, \mathrm{CH})$, $5.46(\mathrm{t}, J=5.2 \mathrm{~Hz}, 1 \mathrm{H}, \mathrm{OH}), 6.07 \sim 6.10(\mathrm{~m}, 1 \mathrm{H}, \mathrm{CH}), 7.55$ $(\mathrm{d}, J=15.2 \mathrm{~Hz}, 1 \mathrm{H}, \mathrm{CH}), 7.59 \sim 7.64(\mathrm{~m}, 1 \mathrm{H}, \mathrm{ArH}), 7.74$ (d, $J=8.4 \mathrm{~Hz}, 1 \mathrm{H}, \mathrm{ArH}), 7.90 \sim 7.93(\mathrm{~m}, 2 \mathrm{H}, 2 \mathrm{ArH}), 8.03$ (d, $J=15.6 \mathrm{~Hz}, 1 \mathrm{H}, \mathrm{CH}), 8.59$ (s, 1H, CH), 11.79 (s, 1H, $\mathrm{NH}) ;{ }^{13} \mathrm{C}$ NMR (DMSO- $\left.d_{6}, 100 \mathrm{MHz}\right) \delta: 37.4,59.3,60.6$, $85.1,85.2,109.1,120.1,127.2,128.0,131.5,133.1,134.3$, 139.3, 140.2, 145.5, 149.7, 162.3, 188.6; IR (KBr) v: 3445, $2105,1717,1686,1596,1581,1466,1293,1098,715 \mathrm{~cm}^{-}$
1. HRMS calcd for $\mathrm{C}_{18} \mathrm{H}_{17} \mathrm{ClN}_{5} \mathrm{O}_{5}[\mathrm{M}+\mathrm{H}]^{+}$: 418.0918, found 418.0931 .

5-(2-苯甲酰基乙烯基)-尿嘧啶核苷(3m): 无色固体, m.p. $156 \sim 157{ }^{\circ} \mathrm{C} ;[\alpha]_{\mathrm{D}}^{22}-45.1$ (c 0.10 , EtOH); ${ }^{1} \mathrm{H}$ NMR $\left(\mathrm{DMSO}-d_{6}, 400 \mathrm{MHz}\right) \delta: 3.60 \sim 3.63(\mathrm{~m}, 1 \mathrm{H}$, $\left.\mathrm{CH}_{2}-1\right), 3.73 \sim 3.76\left(\mathrm{~m}, 1 \mathrm{H}, \mathrm{CH}_{2}-2\right), 3.87 \sim 3.89(\mathrm{~m}, 1 \mathrm{H}$, $\mathrm{CH}), 4.01 \sim 4.04(\mathrm{~m}, 1 \mathrm{H}, \mathrm{CH}), 4.08 \sim 4.10(\mathrm{~m}, 1 \mathrm{H}, \mathrm{CH})$, $5.09(\mathrm{~d}, J=5.6 \mathrm{~Hz}, 1 \mathrm{H}, \mathrm{OH}), 5.38(\mathrm{t}, J=5.2 \mathrm{~Hz}, 1 \mathrm{H}, \mathrm{OH})$, $5.48(\mathrm{~d}, J=5.2 \mathrm{~Hz}, 1 \mathrm{H}, \mathrm{OH}), 5.76(\mathrm{~d}, J=4.0 \mathrm{~Hz}, 1 \mathrm{H}, \mathrm{CH})$, 7.47 (d, $J=15.2 \mathrm{~Hz}, 1 \mathrm{H}, \mathrm{CH}), 7.55$ (t, $J=7.6 \mathrm{~Hz}, 2 \mathrm{H}$, 2ArH), 7.64 (t, $J=7.2 \mathrm{~Hz}, 1 \mathrm{H}, \operatorname{ArH}), 7.93$ (d, $J=7.2 \mathrm{~Hz}$, 2H, 2ArH), 8.03 (d, $J=15.2 \mathrm{~Hz}, 1 \mathrm{H}, \mathrm{CH}), 8.66$ (s, 1H, $\mathrm{CH}), 11.73(\mathrm{~s}, 1 \mathrm{H}, \mathrm{NH}) ;{ }^{13} \mathrm{C}$ NMR (DMSO- $d_{6}, 100 \mathrm{MHz}$ ) $\delta: 60.5,69.3,74.4,85.0,89.3,109.2,120.6,128.5,129.3$, 133.4, 138.0, 138.2, 144.9, 149.9, 162.3, 189.8; IR (KBr) $v: 3398,1689,1594,1467,1292,1100 \mathrm{~cm}^{-1}$. HRMS calcd for $\mathrm{C}_{18} \mathrm{H}_{19} \mathrm{~N}_{2} \mathrm{O}_{7}[\mathrm{M}+\mathrm{H}]^{+}: 375.1192$, found 375.1184 .

5-[2-(4-氯苯甲酰基)乙烯基]-尿嘧啶核苷(3n): 无色 固体, m.p. $217 \sim 218{ }^{\circ} \mathrm{C}$; $[\alpha]_{\mathrm{D}}^{22}-96.3$ (c $\left.0.10, \mathrm{EtOH}\right)$; ${ }^{1} \mathrm{H}$ NMR (DMSO- $\left.d_{6}, 400 \mathrm{MHz}\right) \delta: 3.59 \sim 3.63(\mathrm{~m}, 1 \mathrm{H}$, $\left.\mathrm{CH}_{2}-1\right), 3.73 \sim 3.77\left(\mathrm{~m}, 1 \mathrm{H}, \mathrm{CH}_{2}-2\right), 3.87 \sim 3.88(\mathrm{~m}, 1 \mathrm{H}$, $\mathrm{CH}), 4.01 \sim 4.10(\mathrm{~m}, 2 \mathrm{H}, \mathrm{CH} \times 2), 5.07(\mathrm{~d}, J=5.6 \mathrm{~Hz}, 1 \mathrm{H}$, $\mathrm{OH}), 5.35(\mathrm{t}, J=4.4 \mathrm{~Hz}, 1 \mathrm{H}, \mathrm{OH}), 5.47(\mathrm{~d}, J=4.8 \mathrm{~Hz}, 1 \mathrm{H}$, $\mathrm{OH}), 5.76(\mathrm{~d}, J=3.6 \mathrm{~Hz}, 1 \mathrm{H}, \mathrm{CH}), 7.48$ (d, $J=15.6 \mathrm{~Hz}$, $1 \mathrm{H}, \mathrm{CH}), 7.61$ (d, $J=8.4 \mathrm{~Hz}, 2 \mathrm{H}, 2 \mathrm{ArH}), 7.94$ (d, $J=8.0$ $\mathrm{Hz}, 2 \mathrm{H}, 2 \mathrm{ArH}), 8.00$ (d, $J=15.6 \mathrm{~Hz}, 1 \mathrm{H}, \mathrm{CH}), 8.66$ (s, 1H, $\mathrm{CH}), 11.72$ (br s, $1 \mathrm{H}, \mathrm{NH}) ;{ }^{13} \mathrm{C}$ NMR (DMSO- $d_{6}, 100$ MHz) $\delta: 60.4,69.3,74.4,85.0,89.3,109.1,120.2,129.4$, $130.4,136.8,138.3,138.6,145.1,149.9,162.3,188.7$; IR (KBr) v: 3379, 1691, 1593, 1467, 1298, 1094, $833 \mathrm{~cm}^{-1}$. HRMS calcd for $\mathrm{C}_{18} \mathrm{H}_{18} \mathrm{ClN}_{2} \mathrm{O}_{7}[\mathrm{M}+\mathrm{H}]^{+}:$409.0803, found 409.0814 .

辅助材料(Supporting Information) 化合物 $\mathbf{3 a} \sim \mathbf{3 n}$ 的 ${ }^{1} \mathrm{H}$ NMR 及 ${ }^{13} \mathrm{C}$ NMR 图谱. 这些材料可以免费从本刊网 站(http://sioc-journal. cn/)上下载.

\section{References}

[1] (a) Chappuis, F.; Sundar, S.; Hailu, A.; Ghalib, H.; Rijal, S.; Peeling, R. W.; Alvar, J.; Boelaer, M. Nat. Rev. Microbiol. 2007, 5, 873.

(b) Murray, H. W.; Berman, J. D.; Davies, C. R.; Saravia, N. G. Lancet 2005, 366, 1561.

[2] WHO, Report of a Meeting of the WHO Expert Committee on the Control of the Leishmaniases, Geneva, 2010.

[3] (a) Herwaldt, B. L. Lancet 1999, 354, 1191.

(b) Jha, T. K. Indian J. Med. Res. 2006, 123, 389.

(c) Sundar, S.; More, D. K.; Singh, M. K.; Singh, V. P.; Sharma, S.; 
Makharia, A.; Kumar, P. C.; Murray, H. W. Clin. Infect. Dis. 2000, 31,1104 .

(d) Freitas-Junior, L. H.; Chatelin, E.; Kim, H. A. Siqueira-Neto, J.

L. Int. J. Parasitol:: Drugs Drug Resist. 2012, 2, 11.

[4] (a) Jordheim, L. P.; Durantel, D.; Zoulim, F.; Dumontet, C. Nat. Rev. Drug Discovery 2013, 12, 447.

(b) Wainberg, M. A. Antiviral Res. 2009, 81, 1.

(c) Jin, X; Xiao, Q.; Ju, Y. Chin. J. Org. Chem. 2009, 29, 44 (in Chinese).

(靳玄烨, 肖强, 巨勇, 有机化学, 2009, 29, 44.)

(d) Menéndez-Arias, L.; Álvarez, M.; Pacheco, B. Curr. Opin. Virol. 2014, 8,1 .

(e) Zhang, X..; Wang, A.; Li, D.; Xu, Y. Prog. Chem. 2010, 22, 784 (in Chinese).

(张晓辉, 王爱玲, 李德鹏, 徐耀忠, 化学进展, 2010, 22, 784.)

[5] (a) Xia, Y.; Liu, Y.; Rocchi, P.; Wang, M.; Fan, Y.; Qu, F.; Iovanna, J. L.; Peng, L. Cancer Lett. 2012, 318, 145.

(b) Wu, J.; Yu, W.; Fu, L.; He, W.; Wang, Y.; Chai, B.; Song, C.;
Chang, J. Eur. J. Med. Chem. 2013, 63, 739.

(c) Shirouzu, H.; Morita, H.; Tsukamoto, M. Tetrahedron 2014, 70, 3635 .

[6] (a) Shin, I. S.; Tanifuji, H.; Arata, Y.; Morizawa, Y.; Nakayama, T.; Wataya, Y. Parasitol. Res. 1995, 81, 622.

(b) Peyron, C.; Benhida, R.; Bories, C.; Loiseau, P. M. Bioorg. Chem. 2005, 33, 439.

[7] (a) Torrence, P. F.; Fan, X.; Zhang, X.; Loiseau, P. M. Bioorg. Med. Chem. Lett. 2006, 16, 5047.

(b) Fan, X.; Feng, D.; Qu, Y.; Zhang, X.; Wang, J.; Loiseau, P. M.; Andrei, G.; Snoeck, R.; De Clercq, E. Bioorg. Med. Chem. Lett. 2010, 20, 809 .

[8] Boeck, P.; Falcao C. A. B.; Leal, P. C.; Yunes, R. A.; Cechinel, V.; Torres-Santos, E. C.; Rossi-Bergmann, B. Bioorg. Med. Chem. 2006, 14, 1538.

[9] Liu, M.; Wilairat, P.; Croft, S. L.; Tan, A. L.-C.; Go, M.-L. Bioorg. Med. Chem. 2003, 11, 2729. 